\title{
Improving Control over Inter ISP Traffic Volume and Enhancing Peer Selection Methodology
}

\author{
Mudassar Nawaz \\ ICT, UET Peshawar \\ Pakistan
}

\author{
Aftab Hussain, PhD \\ Senior Manager, PTCL \\ Pakistan
}

\author{
Faisal Shahzad \\ NUST, Islamabad \\ Pakistan
}

\begin{abstract}
Modern day internet communication has evolved due to advance application layers software. These application layer software use distinct protocols like TCP, HTTP and SMTP, etc. to carry out unicast, broadcast and non-broadcast (multicast) network protocols in order to meet customers' demand. Then there are peer to peer and non-peer to peer services. During recent times, peer to peer communication has grabbed a huge chunk of internet communication which engages a lot of network resources. Downloading and uploading with peer to peer connectivity has become a major problem for ISPs to handle. They simply choke networks and especially cross ISP links. In the wake of these problems, ISPs have enabled their systems with certain policies which are translated as limitations. Technologists and engineers have formulated applications that cooperate with ISPs to establish a harmony by not exceeding the limits. Yet there is a huge demand by ISPs to make software developers cognizant of network constraints during software development. Most of the techniques use a collaborative mechanism in which signaling between ISP and End system takes place for the purpose. However these techniques have their scenarios in which they work. This research makes use of multiple limitation techniques to bring down the inter-ISP traffic transpiring a reduction in cost. The ISP-Application resources are the same but a hybrid and dynamic system is attempted in this research to reduce traffic and cost along with helping peers with better experience.
\end{abstract}

\section{Keywords}

peer to peer; $\mathrm{P} 2 \mathrm{P}$

\section{INTRODUCTION}

Initially, most Internet services were asymmetric in nature, with low-capacity clients invoke services from high-capacity servers owned and operated by ISP. However, as the cost for access bandwidth and computation resources decreased, the Internet connected hosts became increasingly powerful. As a consequence, new Internet services arose that shifted away from this model and moves towards a much more usercentered model. First enterprises and then individuals started deploying their own servers, thus making the network edge more and more important for the service delivery. However, the limitations of client/server systems become obvious in large scale distributed environment. In such systems individual resources are concentrated on one or a small number of nodes and in order to provide access with acceptable response time, sophisticated load balancing and fault-tolerance algorithms have to be applied. Moreover the limitation on the network bandwidth adds the bottleneck problem.

These problems have motivated researchers to come up with approaches to distribute processing loads and network bandwidth among all nodes participating in a distributed system. This continuing trend persists today in the shape of massively distributed; global-scale systems based on peer-topeer (P2P) systems. The techniques on which these systems are based have led to many innovations in distributed systems theory and practice, particularly in regards to overlay creation, content search, distribution, replication and quality adaptation.

P2P systems are distributed Internet system in which peers cooperate with each other's to achieve a desired service. There are several concepts underlying P2P systems, like sharing resources, decentralization and self-organization.

- Resource sharing implies that peers participate in the distribution and consumption of resources. Shared resources can be physical resource (disk space, processing power or network bandwidth) as well as logical resources such as services or different forms of knowledge.

- Decentralization is an immediate consequence of sharing resources. P2P systems offer the possibility to distribute video content to an unlimited number of users and reducing the bandwidth bottleneck on the source. The use of centralized server architecture is not needed since the content distribution throughout the network is handled by each peer. It is particularly interesting in order to avoid single point of failures.

- Peers have to self-organize themselves based on the available local information and interaction with other peers. The global behavior then emerges as the result of all the local behaviors results in a pure P2P system.

P2P networking has emerged as a viable business and systems architecture for Internet-scale applications. Although its technological roots trace back through several decades of designing distributed information systems, but $\mathrm{P} 2 \mathrm{P}$ provides an effective way to build applications that connect millions of users across the globe without reliance on specially deployed servers. Instead, by combining the resources of each user's computer, these systems automatically self-organize and adapt to changing peer populations while providing services for content sharing and delivery.

$\mathrm{P} 2 \mathrm{P}$ is one the major and popular application over the internet now days. People use P2P software for file downloading and for multimedia streaming. Due to popularity of $\mathrm{P} 2 \mathrm{P}$ applications demand for high speed internet connection has also been increased. P2P software produces lot of traffic and thus occupies major portion of the internet backbone bandwidth. More utilization of internet bandwidth increases the revenue of ISPs, but on the other hand, high consumption of bandwidth due to $\mathrm{P} 2 \mathrm{P}$ traffic has resulted in degraded 
performance of other traditional and important internet applications like web traffic.

Infrastructure cost for ISP has been increased due to high bandwidth demand, which resulted in installation of advanced hardware. Operational cost has also been increased due to addition of complexity like traffic shaping policies to overcome above mentioned $\mathrm{P} 2 \mathrm{P}$ problems.

P2P system uses its own overlay network, remains unaware of underlay network topology and traffic engineering policies. They select peers for downloading contents randomly instead of selecting peer which is nearest and has optimal route. This nature of P2P system causes huge amount of cross-ISP traffic flow which is another expense at ISP end.

Due to above issues ISPs has started considering P2P as unimportant traffic, which has no major impact on revenue generation. To handle this issue, initially ISPs implemented traffic shaping policies when $\mathrm{P} 2 \mathrm{P}$ ran on standard range of ports. But P2P systems started using nonstandard and random ports, which were difficult for ISPs to detect. So ISPs started using deep packet inspection by installing advanced hardware for detection and shaping of P2P traffic. Again P2P clients bypassed this barrier by encrypting their traffic. Another choice was to use the cache servers at ISP end so that cross ISP traffic can be reduced. By doing so ISP may become a part of distributing illegal and copy righted material. It also violates the law by transferring non transparent traffic between peers. Resetting P2P connections using firewall at ISP level has also legality issues. So crux of this discussion is that we cannot rely exclusively on ISP to address P2P traffic engineering issues.

P2P software are used for file and multimedia downloading. Kaza, lime wire, Emule are the examples of P2P software. Bittorent is the most popular P2P software due to its high performance.

For uploading a file in bittorent, we need to create a torrent file, which is a small file and contains metadata of original file. This file is made available to other P2P users through bittorent, and the peer (node) sharing this complete file is known as seeder. Peers, who want to download a file, need to provide that torrent file to their bittorent application. Peer which downloads a file is known as leech node. Group of Peers which are sharing same metadata contents are called swarm of hosts. Tracker servers assist in identification of peers for downloading a particular file. Tracker keeps list of all peers which contain that specific file. Initially node connects with random no. of peers, for downloading file, but during transfer of file if some peers are not providing enough download rates then those peer connections are dropped (choked) and new peers are selected from list.

P2P creates its own overlay network, i.e. It considers all the peers involved in file sharing as network nodes, and selects random peers instead of selecting nearest peer. For example Peer located in Pakistan may select another Peer located in USA for downloading file. However there may exist Peer sharing same file in Pakistan. This Inefficient behavior of P2P causes lot of Cross-ISP traffic, thus increasing burden on ISP in terms of financial and operational overheads. 'P2P traffic share is almost $70 \%$ of internet bandwidth [22], which results in choking of other internet traffic.

To alleviate this issue ISPs has already taken different measures in past like bandwidth limiting, shaping of traffic and caching of bittorent traffic. But all these single sided approaches were not proved to be effective. Some collaborative models were suggested by different people to handle these tasks effectively.

\section{LITERATURE REVIEW}

Through various researches, discussed below, it has been pointed out that ISP-P2P cooperation have three classes that can reduce inter AS traffic. They are ISP driven, Peer Driven (Application) and caching method [18]. Peer driven method influences on the neighbor peer selection [19] it uses latency metric to find out proximal peer. Another method was introduced that used AS topology which helped in inter traffic reduction [20]. The main aim therefore becomes to make P2P systems more ISP friendly. The overlay construction (path) is influenced with the help of application layer decisions on peers [21]. A typical method which uses caching (with the help of separate storage servers) of P2P contents [22] helps in reducing access to other ISPs every time. The paths are redirected and cache is enabled to act as image of the destination node.

Other methods include optimization of network traffic by enabling application layer traffic optimization [23]. This methodology is known as ALTO. The peer application layer applies optimization procedure to avoid inter ISP traffic. This helps in achieving higher download speed. This work [24] was proposed initially in 2006 [25]. The application takes up a list of ISPs and prioritizes its own ISP. This method has another name as 'biased neighbor selection'. In our generic simulation we will see that a priority set will increase the biasedness of home ISP which will help in reducing the crossing incidents.

Another method uses content distribution network [26]. CDN is made as reference in order to find out proximity of two peers. The contents are distributed within an ISP and the contents are accessed simultaneously. The topology information is sent and noted by Peer Application and the access to other peer is made by calculating the distribution network. Main issue with this type of system was the overburdening of CDN system. In an advancement made [27] ISP is entrusted with the task to provide nearby information to a peer. In this method nearby information is added with the network state information which is indeed of great value [28]. A cooperation mechanism between ISP and P2P systems is important but some $\mathrm{P} 2 \mathrm{P}$ software does not cooperate due to privacy and dynamic port usage utility.

One of the most popular solutions was the installation of Oracle Server [19] to help choosing peer node. The peer application software finds feasible peers. This phenomenon is just like the calculation of feasible successor and successor (Administrative Distance and Feasible Distance in EIGRP). The list of peers is provided to the ISP maintained Oracle (database) Server. Oracle Systems are empowered with sorting function which processes the database provided to it on its own. Oracle system is configured with local/ ISP strategy and policies. The list of peers which comes into its data base from a requesting-peer is sorted and sent back to the peer application. Following priority metrics can be used for the purpose of sorting
a. Geographically
b. Bandwidth calculations
c. Load and delay
d. ISP charging and policies etc. 
Simulations have suggested that this method reduces bandwidth wastage along with reduction in Inter ISP traffic and increase in download performance. The Oracle data base actually aids for choosing peer. It also helps in reduction leeching which is detrimental for download performance.

\section{SUGGESTED SOLUTION}

Cross Network traffic reduction has been successfully attained in works by Sheng [30] and Vinay [19]. The performance has been correspondingly improved keeping in view parameters of data throughput and quality in previous chapters. Sheng proposed a model in which localized selection of peers was used and data transmission was restricted to neighbors/ proximity. Model which Vinay proposed for peer to peer corporate users in order to improve performance, proved to be successful.

An autonomous system (ISP) has better information about the underlay network parameters. Main information includes Bandwidth, distance, policies, geographical location etc. ISP also has oracle based services for the purpose of ranking. P2P services in a network are provided with list of random peers and then a ranking is done. The issue of trust between P2Pand ISP is maintained with the help of ranking algorithm however $\mathrm{P} 2 \mathrm{P}$ can be manipulated by ISP (favored).

Smallest RTT first method proposed by Sheng used ranking mechanism proposed on the base of calculation of RTT. Nearest peer is selected on the base of two indicators k-r. Where, ' $\mathrm{k}$ ' is the total number of peers provided by the tracker and ' $r$ ' is the random integer. This method does not require additional server like Oracle. The issue in this method was latency. Other issues are related to multiple AS problem in a single ISP that lowers the performance. Some other previously suggested solutions include $\mathrm{P} 4 \mathrm{P}$ provider portal. The Oracle servicer is provided by ISP or third party. In this method ISP can trick by hiding true information.

The model proposed a ranking method which keeps in view following parameters:
a. RTT between peers
b. Low latency RTT prioritization
c. AS labeling of peers

The solution suggests that an aggregate of the results of the two of the aforementioned solutions should be made (Information attained from ISP and P2P). The preference of intra ISP peers will be increased by multiplying it with a factor. Later P2P application generates independent ranked lists which will give above given information. ISP oracle provides independent ranking list. This gives best peer ranking based on bandwidth, delay and number of Hops. A scenario is given in the table 1 . To summarize and objectify the above theory of ranking take example of peers from A to I. RTT result Measured by RTT, from Peer B, D stands for different ISP. For a same ISP, notation of S is being used. The peers contain IPs orderly 111.68.99.129, 182.177.23.92, 182.177.28.95, 58.65.178.108, $\quad 203.99 .62 .13$ 119.154.104.111, 119.154.105.1, 202.147.161.241, 137.138.144.169. The mentioned ISPs are CERN(AS 513), PTCL(AS 17557), HECPERN(AS 45773), TWA(AS 38193), LDN(AS 23966), Nayatel(AS 23674)

\begin{tabular}{|c|c|c|}
\hline PEER & RTT(msec) & TAG \\
\hline A & 64 & D \\
\hline B & X & X \\
\hline C & 62 & S \\
\hline D & 49 & D \\
\hline E & 74 & D \\
\hline F & 139 & S \\
\hline G & 147 & S \\
\hline H & 98 & D \\
\hline I & 334 & D \\
\hline
\end{tabular}

Table 1: Ranking Method

\begin{tabular}{|l|l|}
\hline PEER & RAN N \\
\hline A & 3 \\
\hline B & X \\
\hline C & 2 \\
\hline D & 1 \\
\hline E & 4 \\
\hline F & 6 \\
\hline G & 7 \\
\hline H & 5 \\
\hline I & 8 \\
\hline
\end{tabular}




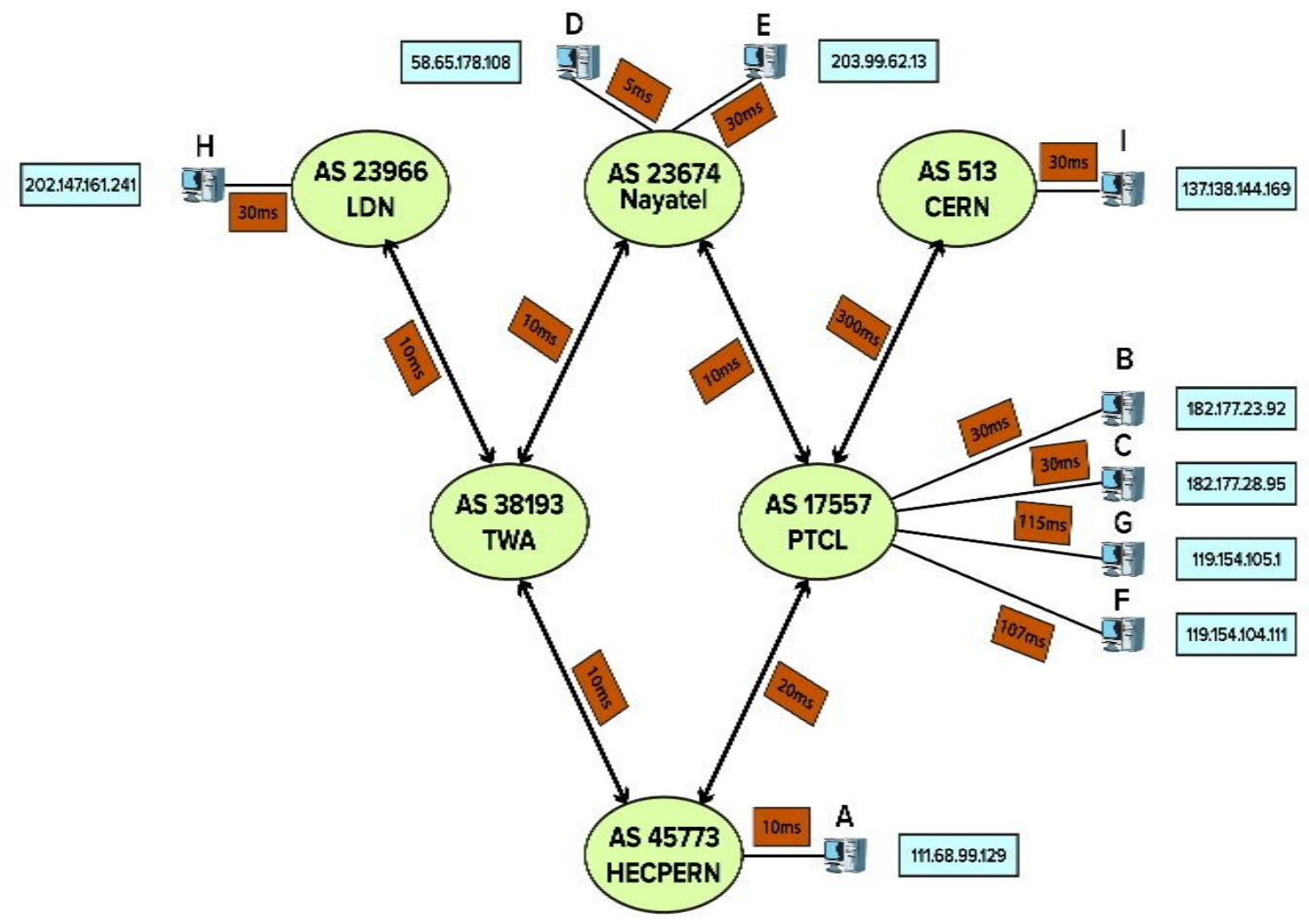

Figure 1: Simulation Scenario

For the above case best case ISP ranking list will be

Table 2: Ranking (Rearrangement through Priority)

\begin{tabular}{|c|c|}
\hline PEER & RANK \\
\hline A & 4 \\
\hline B & X \\
\hline C & 1 \\
\hline D & 6 \\
\hline E & 5 \\
\hline F & 2 \\
\hline G & 3 \\
\hline H & 7 \\
\hline I & 8 \\
\hline
\end{tabular}

Aggregate Results are (Adding the rank value of P2P and ISP and generate accumulated rank- Given Below).
Table 3: Aggregating the results for ranking each peer

\begin{tabular}{|c|c|c|c|c|c|c|c|c|}
\hline PEERS & A & C & D & E & F & G & H & I \\
\hline TAG & D & S & D & D & S & S & D & D \\
\hline P2P Rank & 3 & 2 & 1 & 4 & 6 & 7 & 5 & 8 \\
\hline & + & + & + & + & + & + & + & + \\
\hline ISP Rank & 4 & 1 & 6 & 5 & 2 & 3 & 7 & 8 \\
\hline Total Value & 7 & 3 & 7 & 9 & 8 & 10 & 12 & 16 \\
\hline
\end{tabular}

Note that as proposed in case of same ISP peers Tagged S an increase is given for prioritization by multiplying final rank with 0.3 and decreasing of preference for peer communication by multiplying a factor of 0.7 (where label $\mathrm{D}$ is given). The final ranks will be: 


\begin{tabular}{|l|l|l|l|l|l|l|l|l|}
\hline PEERS & A & C & D & E & F & G & H & I \\
\hline TAG & D & S & D & D & S & S & D & D \\
\hline P2P Rank & 3 & 2 & 1 & 4 & 6 & 7 & 5 & 8 \\
\hline & + & + & + & + & + & + & + & + \\
\hline ISP Rank & 4 & 1 & 6 & 5 & 2 & 3 & 7 & 8 \\
\hline Total Value & 7 & 3 & 7 & 9 & 8 & 10 & 12 & 16 \\
\hline Constant & 0.7 & 0.3 & 0.7 & 0.7 & 0.3 & 0.3 & 0.7 & 0.7 \\
\hline Final Rank & 4.9 & 0.9 & 4.9 & 6.3 & 2.4 & 3 & 8.4 & 11.2 \\
\hline
\end{tabular}

Table 4: Final ranking

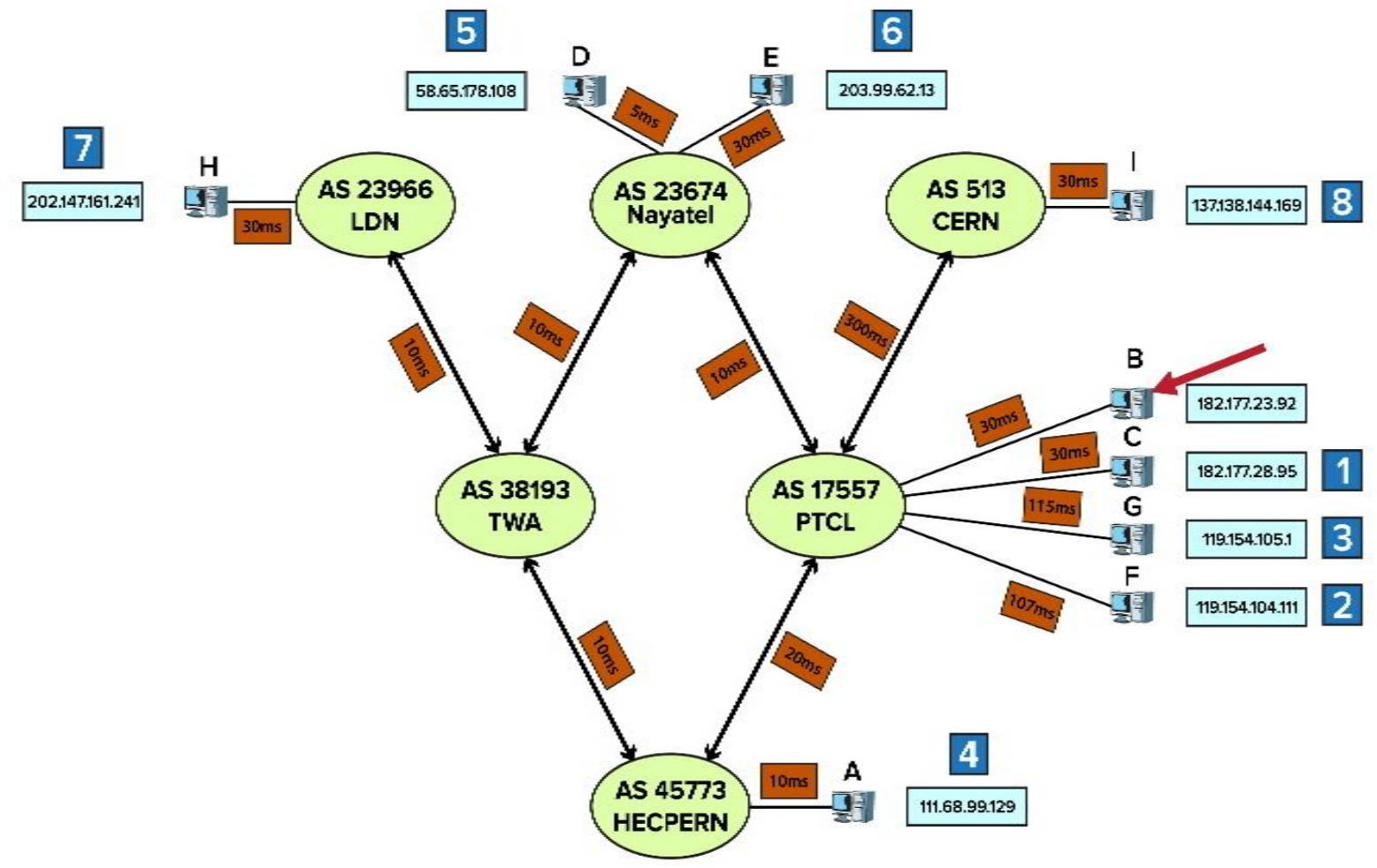

Figure 2: Final Preference based ranking

The above mentioned is best case scenario whereas, in case ISP modifies ranking the modification/ proposed solution has produced still better. The results are given below:

Table 5: Worst case results

\begin{tabular}{|c|c|c|c|c|c|c|c|c|}
\hline PEERS & A & C & D & E & F & G & H & I \\
\hline TAG & D & S & D & D & S & S & D & D \\
\hline P2P Rank & 3 & 2 & 1 & 4 & 6 & 7 & 5 & 8 \\
\hline & + & + & + & + & + & + & + & + \\
\hline ISP Rank & 3 & 6 & 1 & 4 & 5 & 7 & 2 & 8 \\
\hline Total Value & 6 & 8 & 2 & 8 & 12 & 14 & 7 & 16 \\
\hline Constant & 0.7 & 0.3 & 0.7 & 0.7 & 0.3 & 0.3 & 0.7 & 0.7 \\
\hline Final Rank & 4.2 & 2.4 & 1.4 & 5.6 & 3.6 & 4.2 & 4.9 & 11.2 \\
\hline
\end{tabular}




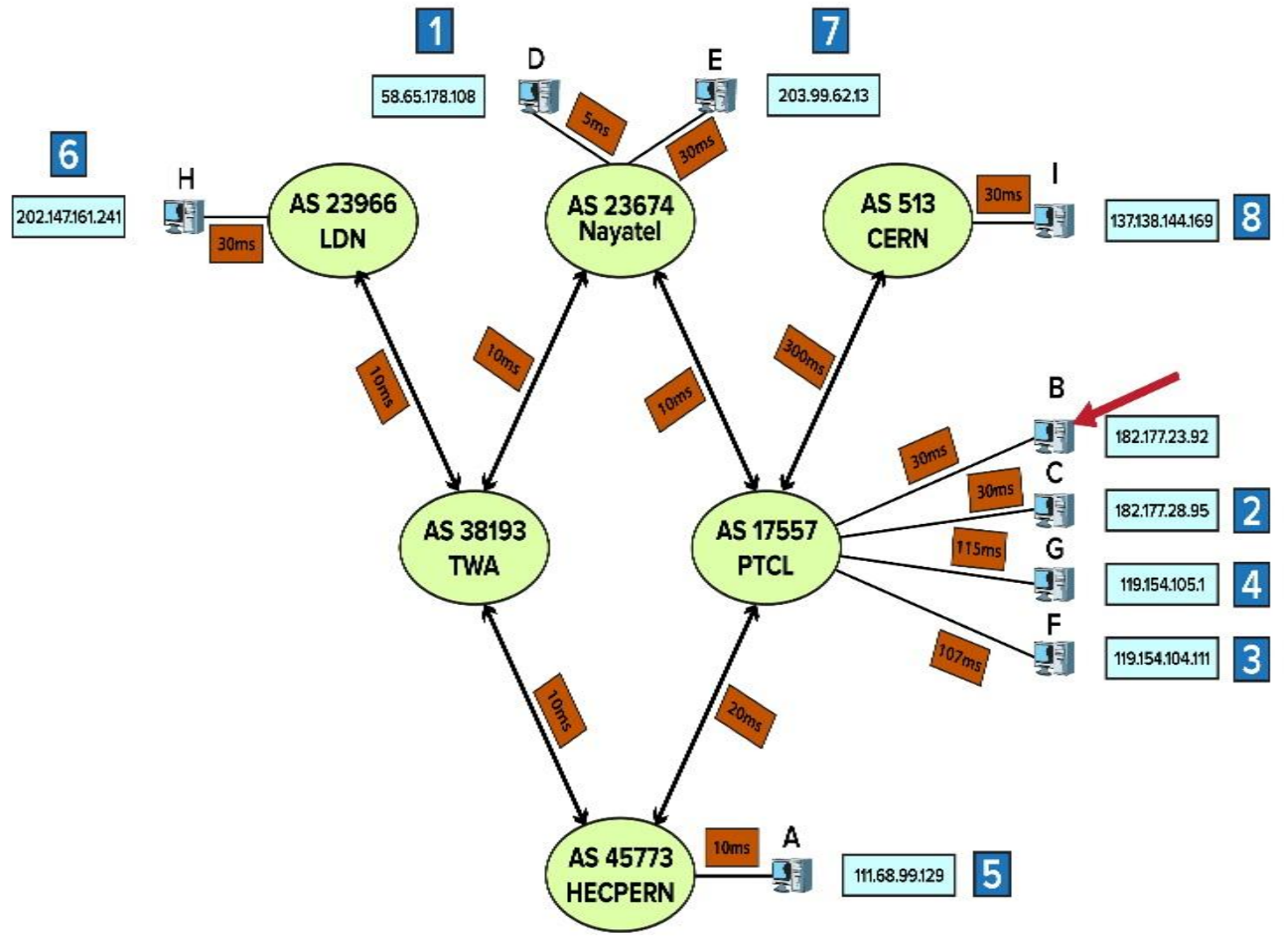

Figure 3: Ranking in worst case scenario

The proposed solution provides credible output and the aggregates the advantages of the above two given scenarios. The whole responsibility is not thrown at the ISP so any lopsidedness shown by the ISP is not effective as in previous cases. The performance is improved while priority method is still simple.

\section{TRAFFIC STATS AND PROGNOSIS}

Best and worst case scenarios in simulation results are given below.

Looking at figure 4.1 first part shows the values aggregated against each peer the second part shows the final ranking of each peer. Remember these final ranking are based upon values aggregated again each peer.

Figure 4.2 computes the same values for the worst case scenario. A conclusion can be drawn from this figure that beyond a certain limit the aggregated value for a peer shows an increasing trend. The resultant ranking of the peer will also increase. This is consistent with both the best case and the worst case scenarios.

Let's consider the case of Fig 4.1 for instance, the aggregated value for peer A is five, it steeps down to zero for peer C. Then there is an increasing trend up till peer E. It again shows a falling trend for peer $\mathrm{F}$ and $\mathrm{G}$. After that there is a sharp increase for peer $\mathrm{H}$ and $\mathrm{I}$. This shows that from peer $\mathrm{A}$ to $\mathrm{E}$, the average aggregated value remains almost constant but for peer $\mathrm{H}$ and $\mathrm{I}$, a sharp increase is noticed, so it will be interesting to see whether or not this trend continues as we add on more peers.
One other observation that can be made seeing these simulation results is that the aggregated ranking method creates a cohesion between ISP and P2P. A single ended control does not work effectively. A hybrid or a collaborative model achieve better results. Also better caching techniques leads to fewer ISP to ISP traffic and hence lesser cost. The quality of service to the end user will also improve.
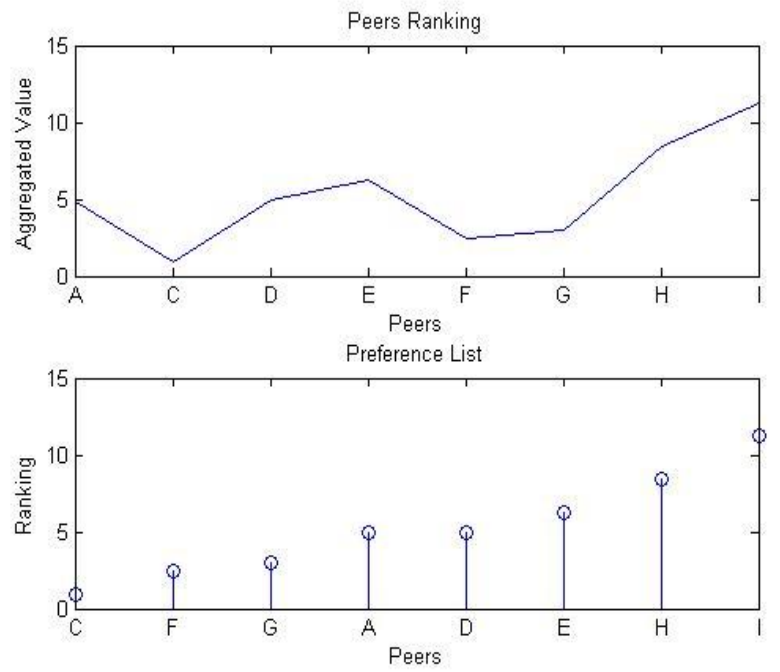

Figure 4.1 Best case Scenario 

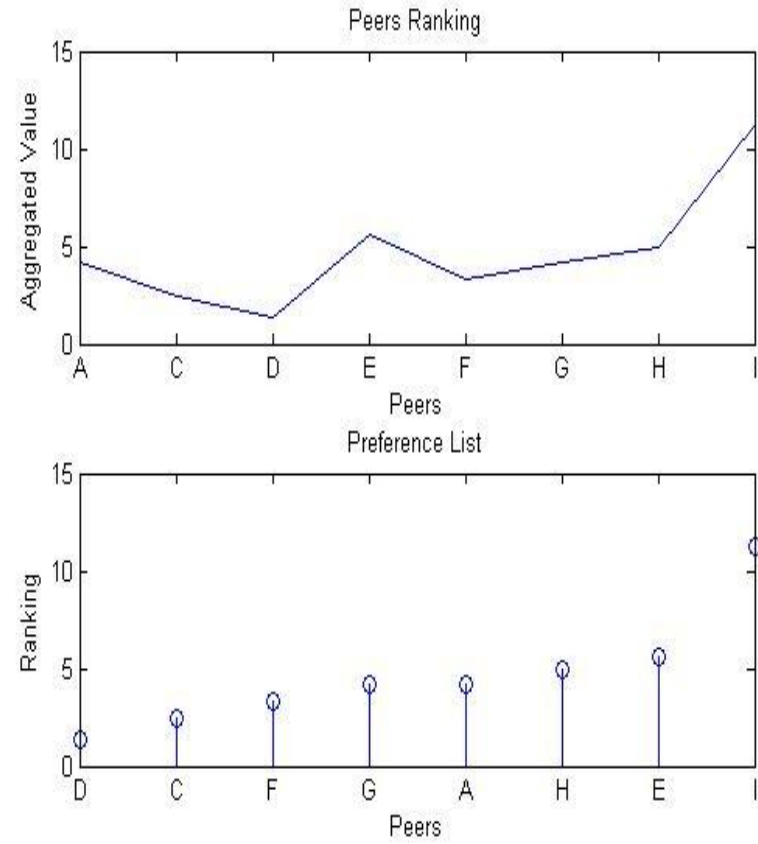

Figure 4.2 Worst case Scenario

\section{CONCLUSION}

A hybrid model for ISP-P2P collaboration in reduction of inter-ISP traffic is new for technologists and network engineers. It is of great value and its ease of implementation comes from the notion that it makes use of already available network resources. Different techniques have been devised to limit the maximum utilization and cost issues. However there is growing demand from ISPs to check non-P2P traffic. The simultaneous utilization of options has been researched and designed in this thesis which effectively reduces across ISP overlay traffic and cost.

The hybrid model for ISP-P2P collaboration is a new research field with a vast scope for further research. It emphasizes on the optimum utilization of the available network resources in order to reduce the inter ISP traffic. Which can make the solution more business friendly as well as user friendly. This dissertation is an attempt to probe the benefits of a simultaneous approach in order to enhance the user experience and to reduce the cost. Based on this approach a few facts need to be pondered upon.

1. First of them being the capability of the individual peer should be enhanced using robust algorithms and better caching techniques.

2. This can also be achieved by adding more hardware to the system. If the applications communicate better among themselves then there will be fewer undesired traffic and better quality at the end of user.

\section{FUTURE WORK}

A real time implementation, in addition to simulation results, of this work is important. Application in this thesis has been designed which needs to be interfaced in ISP enabled environment. For future work, policy implementation at ISP level and signaling between ISP and Application must be done. Also that traffic traces and data capturing will help in understanding developmental constraints of this model. Based on the comparative analysis, mathematical computation and research carried, following future directions can be set in this field.

1. A more robust algorithm can be developed in order to manage the resources of the network. An approach which can give direct access to the peers to certain shared resources with undergoing any conflict situation can be an important future research direction.

2. An algorithm for signalling between the applications can also be developed as to when this signalling should be done, and what will be the relative priority of each signal.

3. The effect of increasing the number of peers on the ranking and aggregated values can also be studied.

4. More research can be focused on the role of the ISP and the control they can exercise on the communication.

5. The concept of semiautonomous peers can also be researched upon whereby the control of the ISP can be minimized.

\section{ACKNOWLEDGMENTS}

A large number of people have made possible and contributed to my work on this thesis in different ways. It is a pleasure to convey gratitude to all of them all acknowledgment. Foremost, the support, guidance and encouragement by supervisor Dr. Aftab Hussain Bangash, Mr. Faisal Shahzad and Mr. Asad Ehsan, help to perform at critical stages. Their professional vision and technical skills always proved helpful in problem solving and addressing issue.

\section{REFERENCES}

[1] Camus (2012). Routed Versus Routing Protocols 1. Retrieved May 2014, from http://cpe.rmutt.ac.th/network/images/cn/Chapter\%205\% 20IP\%20Routing.pdf

[2] Editorial (2013, October). Voice over IP 2: the expert view. Retrieved May 2014, from http://www.computerweekly.com/feature/Voice-over-IPthe-expert-view

[3] Bradely (2012, May). Types of Networks - LAN WAN MAN and Area Networks. Retrieved May 2014,from http://compnetworking.about.com/od/basicnetworkingco ncepts/a/network_types.htm

[4] Konstantinos (2010). Challenges in Cooperation between Internet Service Providers and Peer-to-Peer Application. Retrieved from http://www.doc.ic.ac.uk/teaching/distinguishedprojects/2010/k.gkerpinis.pdf

[5] Mitchel (2013, February). HTG Explains: What's the Difference Between Ad-Hoc and Infrastructure Mode? Retrieved from http://www.howtogeek.com/180649/htg-explains-whatsthe-difference-between-ad-hoc-and-infrastructure-mode/

[6] Ammar (2011). HTG Explains: What's the Difference Between Ad-Hoc and Infrastructure Mode? Retrieved from http://www.howtogeek.com/180649/htg-explainswhats-the-difference-between-ad-hoc-and-infrastructuremode/ 6 
[7] Technet Microsoft (2010). Port Assignments for WellKnown Ports . Retrieved from http://technet.microsoft.com/en-us/library/cc959828.aspx

[8] Rouse (2010). What is dynamic port numbers (private port numbers)? - Definition from WhatIs.com. Retrieved from

http://searchnetworking.techtarget.com/definition/dynam ic-port-numbers

[9] Microsoft (2010). The OSI Model's Seven Layers Defined and Functions Explained. Retrieved from http://support.microsoft.com/kb/103884

[10] TCP Guide (2012). The TCP/IP Guide - OSI Reference Model Layer Summary. Retrieved from http://www.tcpipguide.com/free/t_OSIReferenceModelL ayerSummary.htm

[11] IRCB (2008). Routing and Switching 15. Retrieved from http://www.ircbeginner.com/ircinfo/Routing_Article.pdf

[12] Schluting (2012). Networking 101: Understanding RIP Routing. Retrieved from http://www.enterprisenetworkingplanet.com/netsp/article .php/3609151/Networking-101-Understanding-RIPRouting.htm

[13] CISCO (2013). Introduction to EIGRP - Cisco. Retrieved from

http://www.cisco.com/c/en/us/support/docs/ip/enhancedinterior-gateway-routing-protocol-eigrp/13669-1.html

[14] P2P caching - Wikipedia, the free encyclopedia. (n.d.). Retrieved May 16, 2014, from http://en.wikipedia.org/wiki/P2P_caching

[15] Ohio University (2012). Overlay Networks. Retrieved from http://www.cse.ohiostate.edu/hpcs/WWW/HTML/internet-p2p.html

[16] Gyorgy (2008). Cooperative Caching and Relaying Strategies for Peer-to-peer Content Delivery. Retrieved from http://www.cs.toronto.edu/iptps2008/final/48.pdf

[17] Saumitra (2013). ALTO: A Multi Dimensional Peer Selection Problem. Retrieved from http://www.ietf.org/proceedings/73/slides/alto-4.pdf

[18] White Paper, "Management Peer-to-Peer traffic with Cisco Service Control Technology",
http://www.cisco.com/en/US/prod/collateral/ps7045/ps61 29/ps6133/ps6150/prod_white_paper0900aecd8023500d. html

[19] V. Aggarwalet al., "Can ISPs and P2P Users Cooperate for Improved Performance?”, ACM CCR, Vol. 37, No. 3 , pp.

[20] D.R. Choffnes et al., "Taming the Torrent: A Practical Approach to Reducing Cross-ISPTraffic in P2P Systems", ACM CCR, Vol. 38, No. 4, pp. 363-374, Oct. 2008

[21] SopCast (2009), http://www.sopcast.com

[22] György, (2011), Interaction Patterns between P2P Content Distribution Systems and ISPs, Piotr Cholda and Rafal Stankiewicz, AGH University of Science and Technology Ioanna Papafili and George D. Stamoulis, Athens University of Economics and Business

[23] R. Bindal et al., "Improving Traffic Locality in BitTorrent via Biased Neighbor Selection," Proc. 26th IEEE Int'l. Conf. Distributed Computing Systems (ICDCS), 2006, pp. 66-76.

[24] M. Castro et al., "Topology-Aware Routing in Structured Peer-to-Peer Overlay Networks, Tech. Rep. MSRTR2002-82," 2002. Available: citeseer.ist.psu.edu/ castro02topologyaware.html.

[25] Chaojiong, (2010), IEEE, A Dynamic Peer-to-Peer Traffic Limiting Policy for ISP Networks

[26] G. Dán,(2012) “Cache-to-Cache: Could ISPs Cooperate to Decrease Peer-to-Peer Content Distribution Costs?" IEEE Trans. Parallel and Distr. Syst., to appear

[27] M. Merrit, D. Pasko, and L. Popkin,(2008) "NetworkFriendly Peer-to-Peer Services," Proc. IETF Wksp. P2P Infrastructures, May 2008.

[28] L. Qiu et al., "On Selfish Routing in Internet-Like Environments" Proc. ACM SIGCOMM 2003

[29] H. Xie et al., "P4P: Provider Portal for Applications", Proc. ACM SIGCOMM 2008

[30] Lijie Sheng "Reducing Cross-Network Traffic in P2P Systems via Localized Neighbor Selection" 10.1109/CHINACOM.2009. 